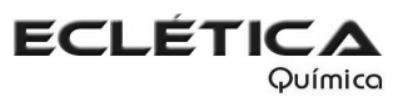

www.scielo.br/eq

www.ecletica.iq.unesp.br

Volume 33, número 4, 2008

\title{
Thermodynamic quantities of solvation and dilution for some acetanilide derivatives in octanol and water mutually saturated
}

\author{
Y. Baena, H. Barbosa, and F. Martínez* \\ Sección de Farmacotecnia, Departamento de Farmacia, Universidad Nacional de Colombia, A.A. \\ 14490, Bogotá D.C., Colombia \\ *fmartinezr@unal.edu.co
}

\begin{abstract}
Based on published thermodynamic quantities for solution, partitioning and sublimation of acetanilide (ACN), acetaminophen (ACP) and Phenacetin (PNC), the thermodynamic quantities for drugs solvation in octanol-saturated water $\left(\mathrm{W}_{(\mathrm{ROH})}\right)$ and water-saturated octanol $\left(\mathrm{ROH}_{(\mathrm{W})}\right)$ as well as the drugs dilution in $\mathrm{ROH}_{(\mathrm{W})}$ were calculated. The Gibbs energies of solvation were favourable in all cases. The respective enthalpies and entropies were negative indicating an enthalpy-driving for the solvation process in all cases. On the other hand, the Gibbs energies of dilution were favourable for ACP and PNC but unfavourable for ACN, whereas the respective enthalpies and entropies were negative for $\mathrm{ACP}$ and PNC but positive for ACN indicating enthalpy-driving for the dilution process in the case of the former drugs and entropy-driving for the latter. From the obtained values for the transfer processes, an interpretation based on solute-solvent interactions was developed.
\end{abstract}

Keywords: Acetanilide derivatives; solvation; partition coefficient; octanol; solution thermodynamics.

\section{Introduction}

Acetaminophen (ACP) is an analgesic and antipyretic drug widely used in modern therapeutics. It is specially indicated in the treatment of several minor diseases presented by pediatric patients [1]. Phenacetin (PNC) was used as analgesic and antipyretic drug long time ago but it was removed from the market because it can induce nephropathy and cancer. On the other hand, acetanilide (ACN) is mainly used as an intermediate in the synthesis of some drugs and dyes [2]. These compounds have a good molecular similarity among them as can be seen in Table 1 .

On the other hand, as useful information in medicinal chemistry, the thermodynamics of transfer of drug compounds can be studied by measuring the partition coefficient and/or solubility as a function of temperature. Such data can be used for the prediction of absorption, membrane permeability, and in vivo drug distribution [3].

Semi-polar solvents have been found to yield better correlations with partitioning of solutes obtained in model membranes compared to non-polar solvents such as cyclohexane $(\mathrm{CH})$, which interacts only by non-specific forces (London interactions). In particular, octanol $(\mathrm{ROH})$ has been found to be a useful solvent as the reference for extrathermodynamic studies in a variety of systems [4].

As a contribution to systematization of physicochemical information about anti-inflamma- 
tory drugs' transfer properties, the main goal of this study was to analyze the solvation and dilution behavior of ACN, ACP, and PNC in the octanol/water $(\mathrm{ROH} / \mathrm{W})$ system by employing a thermodynamic approach based on solubility [5], partitioning [6, 7] and sublimation processes [2]. From the obtained values of the corresponding thermodynamic quantities for drug's transfer processes, an interpretation based on solute-solvent and solvent-solvent interactions was developed.

\section{Theoretical}

The partition coefficient expressed in molality $\left(K_{o / w}^{m}\right)$, for any solute between organic and aqueous phases is calculated by means of:

$$
K_{o / w}^{m}=W_{w} \frac{C_{1}-C_{2}}{C_{2} W_{o}}
$$

where, $W_{w}$ and $W_{o}$ are the masses (usually in g) of aqueous and organic phases, respectively, and $C_{1}$ and $C_{2}$ are the aqueous concentrations of solute (usually in $\mu \mathrm{g} \mathrm{mL}^{-1}$ ) before and after the transfer of the solute from the aqueous phase to the organic medium, respectively [3]. The rational partition coefficients $\left(K_{o / w}^{X}\right.$, , in mole fraction) are calculated from $K_{o / w}^{m}$ values as:

$$
K_{o / w}^{X}=K_{o / w}^{m}\left(M_{o} / M_{w}\right)
$$

where, $M_{o}$ and $M_{w}$ are the molar masses of the organic and aqueous phases, respectively [8].

The standard change for Gibbs free energy of transfer of a solute from an aqueous phase to an organic medium is calculated as follows:

$$
\Delta G_{w \rightarrow o}^{0 X}=-R T \ln K_{o / w}^{X}
$$

On the other hand, the enthalpy change for the transfer may be obtained indirectly by means of the analysis of the temperature-dependence for partitioning by using the van't Hoff method. This procedure permits to obtain the standard enthalpy change ( $\left.K_{o / w}^{m}\right)$ from:

$$
\left(\frac{\partial \ln K_{o / w}^{X}}{\partial(1 / T)}\right)_{P}=-\frac{\Delta H_{w \rightarrow o}^{o X}}{R}
$$

Therefore, $\Delta H_{w \rightarrow o}^{0 X}$ is determined from the slope of a weighted linear plot of $\ln K_{o / w}^{X}$ as a function of $1 / T$. The standard entropy change of transfer is obtained by means of:
$\Delta S_{w \rightarrow o}^{0 X}=\frac{\Delta H_{w \rightarrow o}^{0 X}-\Delta G_{w \rightarrow o}^{0 X}}{T}$

The thermodynamic functions $\Delta H_{w \rightarrow o}^{0 X}$ and $\Delta S_{w \rightarrow o}^{0 X}$ represent the standard changes in enthalpy and entropy, respectively, when one mole of drug is transferred from the aqueous medium to the organic system at infinite dilution expressed in the mole fraction scale [3].

On the other hand, for the solution process of drugs some equations similar to 3,4 and 5 have been used for calculating the respective thermodynamic functions. In this case, drug solubility expressed in mole fraction $\left(X_{2}\right)$ is used instead of $K_{o / w}^{X}[5]$.

\section{Results and Discussion}

All the experimental values of solubility, partitioning and sublimation for the evaluated drugs have been taken from the literature [2, 5-7]. The molecular structure and some physicochemical properties of the drugs are summarized in Table $1[2,9]$. The solubility in water and the $\mathrm{ROH} / \mathrm{W}$ partitioning was determined at $\mathrm{pH} 7.4$ (resembling the blood physiological value). At this $\mathrm{pH}$ value the three compounds are present mainly in their molecular form without dissociation and therefore they have their lowest aqueous solubility and highest partitioning.

Table 1. Some physicochemical properties of the drugs studied.

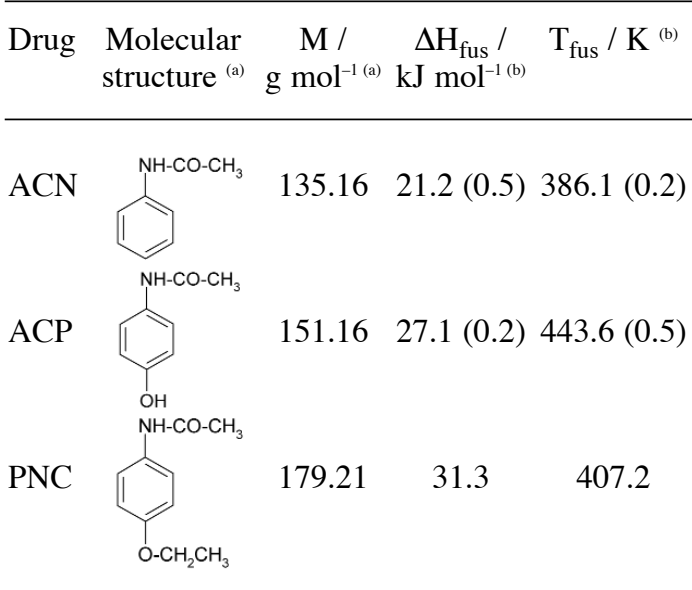

${ }^{(a)}$ From Budavari et al. [9]; ${ }^{(b)}$ From Perlovich et al. [2]. 
Thermodynamics of solution and solvation at saturation

Table 2 summarizes the thermodynamic functions relative to solution processes in octanol-saturated water $\left(\mathrm{W}_{(\mathrm{ROH})}\right)$ and in watersaturated octanol $\left(\mathrm{ROH}_{(\mathrm{W})}\right)$ taken from Ref. [5]. hand, with the aim to compare the relative contributions by enthalpy $\left(\% \zeta_{H}\right)$ and entropy $\left(\% \zeta_{T S}\right)$ toward the solvation process, the equations 9 and 10 were employed.

$$
\% \zeta_{I I}=100 \frac{\left|\Delta H_{\mathrm{solv}}^{0 X}\right|}{\left|\Delta H_{\mathrm{solv}}^{0 X}\right|+\left|T \Delta S_{\mathrm{solv}}^{0 X}\right|}
$$

Table 2. Thermodynamic quantities for drugs solution processes in the aqueous and organic media at $25.0^{\circ} \mathrm{C}^{(\text {a) }}$.

\begin{tabular}{lccccc}
\hline Drug & Solvent & $\begin{array}{c}\Delta G_{\text {soln }}^{0} / \\
\mathrm{kJ} \mathrm{mol}^{-1}\end{array}$ & $\begin{array}{c}\Delta H_{\text {soln }}^{0} / \\
\mathrm{kJ} \mathrm{mol}^{-1}\end{array}$ & $\begin{array}{c}\Delta S_{\text {soln }}^{0} / \\
\mathrm{kJ} \mathrm{mol}^{-1}\end{array}$ & $\begin{array}{c}T \Delta S_{\text {soln }}^{0} / \\
\mathrm{kJ} \mathrm{mol}^{-1}\end{array}$ \\
\hline \multirow{2}{*}{$\mathrm{ACN}$} & $\mathrm{W}_{(\mathrm{ROH})}$ & $17.84(0.01)$ & $30.9(1.7)$ & $43.9(2.4)$ & $13.1(0.7)$ \\
& $\mathrm{ROH}_{(\mathrm{W})}$ & $5.15(0.03)$ & $14.8(0.4)$ & $32.4(1.0)$ & $9.7(0.3)$ \\
$\mathrm{ACP}$ & $\mathrm{W}_{(\mathrm{ROH})}$ & $15.74(0.01)$ & $26.9(1.0)$ & $37.6(1.4)$ & $11.2(0.4)$ \\
& $\mathrm{ROH}_{(\mathrm{W})}$ & $8.97(0.07)$ & $11.4(0.9)$ & $8.0(0.6)$ & $2.4(0.2)$ \\
$\mathrm{PNC}$ & $\mathrm{W}_{(\mathrm{ROH})}$ & $22.79(0.04)$ & $22.5(1.5)$ & $-1.0(0.1)$ & $-0.30(0.03)$ \\
& $\mathrm{ROH}_{(\mathrm{W})}$ & $9.76(0.06)$ & $44(4)$ & $114(10)$ & $34.0(3.0)$ \\
\hline
\end{tabular}

(a) From Baena et al. [5].

The solution process may be represented by the following hypothetic stages [10]:

Solute $_{(\text {Solid) }} \rightarrow$ Solute $_{(\text {Vapor })} \rightarrow$ Solute $_{(\text {Solution })}$

Where, the respective partial processes toward the solution are solute sublimation and solvation, which permits to calculate the partial thermodynamic contributions to solution process by means of equations 6 and 7, respectively, while the Gibbs free energy of solvation is calculated by means of Eq. 8:

$$
\begin{aligned}
& \Delta H_{\text {soln }}^{0}=\Delta H_{\text {subl }}^{0}+\Delta H_{\text {solv }}^{0} \\
& \Delta S_{\text {soln }}^{0}=\Delta S_{\text {subl }}^{0}+\Delta S_{\text {solv }}^{0} \\
& \Delta G_{\text {soln }}^{0}=\Delta G_{\text {subl }}^{0}+\Delta G_{\text {solv }}^{0}
\end{aligned}
$$

The $\Delta H_{\text {subl }}^{0}$ values presented in Table 3, which are required for calculate the solvation quantities, were taken from Perlovich et al. [2], and therefore, the function $\Delta H_{\text {solv }}^{0}$ was calculated from $\Delta H_{\text {soln }}^{0}$ values presented in Table 2.

In Table 4 the thermodynamic functions of solvation are presented, while on the other
Table 3. Thermodynamic quantities for drugs sublimation processes at $25.0^{\circ} \mathrm{C}^{(a)}$.

\begin{tabular}{lcccc}
\hline Drug & $\begin{array}{c}\Delta G_{\text {subl }}^{0} / \\
\mathrm{kJ} \mathrm{mol}^{-1}\end{array}$ & $\begin{array}{c}\Delta H_{\text {subl }}^{0} / \\
\mathrm{kJ} \mathrm{mol}^{-1}\end{array}$ & $\begin{array}{c}\Delta S_{\text {subl }}^{0} / \\
\mathrm{J} \mathrm{mol}^{-1} \mathrm{~K}^{-1}\end{array}$ & $\begin{array}{c}T \Delta S_{\text {subl }}^{0} / \\
\mathrm{kJ} \mathrm{mol}^{-1}\end{array}$ \\
\hline $\mathrm{ACN}$ & 40.5 & $99.8(0.8)$ & $197(2)$ & $58.7(0.6)$ \\
$\mathrm{ACP}$ & 60.0 & $117.9(0.7)$ & $190(2)$ & $56.6(0.6)$ \\
PNC & 52.3 & $121.8(0.7)$ & $226(2)$ & $67.4(0.6)$ \\
\hline
\end{tabular}

(a) From Perlovich et al. [2].

$\% \zeta_{T S}=100 \frac{\left|T \Delta S_{\text {solv }}^{0 X}\right|}{\left|\Delta H_{\text {solv }}^{0 X}\right|+\left|T \Delta S_{\text {solv }}^{0 X}\right|}$

From the values of $\% \zeta_{H}$ and $\% \zeta_{T S}$ presented in Table 4 it follows that the main contributing force to standard free energy of the solvation process of these drugs in both solvents is the enthalpy, especially for PNC in $\mathrm{ROH}_{(\mathrm{W})}\left(\% \xi_{H}\right.$ is $\left.70 \%\right)$.

Because not only the main driving force of solvation process of drug compounds is important, but also the balance between specific and non-specific solute-solvent interactions as well, therefore parameters which describe the relative ratio of specific and non-specific solutesolvent interaction in terms of enthalpies $\left(\% \varepsilon_{H}\right)$ 
Table 4. Thermodynamic quantities for drugs solvation processes in the aqueous and organic media at $25.0{ }^{\circ} \mathrm{C}$ obtained by considering the solubility behavior.

\begin{tabular}{lccccccccc}
\hline Drug & Solvent & $\begin{array}{c}\Delta G_{\text {solv }}^{0} / \\
\mathrm{kJ} \mathrm{mol}^{-1}\end{array}$ & $\begin{array}{c}\Delta H_{\text {solv }}^{0} / \\
\mathrm{kJ} \mathrm{mol}^{-1}\end{array}$ & $\begin{array}{c}\Delta S_{\text {solv }}^{0} / \\
\mathrm{J} \mathrm{mol}^{-1} \mathrm{~K}^{-1}\end{array}$ & $\begin{array}{c}T \Delta S_{\text {solv }}^{0} / \\
\mathrm{kJ} \mathrm{mol}^{-1}\end{array}$ & $\% \boldsymbol{\zeta}_{\boldsymbol{H}}$ & $\% \boldsymbol{\xi}_{\boldsymbol{T S}}$ & $\% \varepsilon_{\boldsymbol{H}}$ & $\% \varepsilon_{S}$ \\
\hline \multirow{2}{*}{$\mathrm{ACN}$} & $\mathrm{W}_{(\mathrm{ROH})}$ & -22.7 & $-68.9(1.9)$ & $-153(3)$ & $-45.6(0.9)$ & 60.2 & 39.8 & 123.7 & 72.4 \\
& $\mathrm{ROH}_{(\mathrm{W})}$ & -35.4 & $-85.0(0.9)$ & $-165(2)$ & $-49.1(0.6)$ & 63.4 & 36.6 & 176.0 & 79.6 \\
$\mathrm{ACP}$ & $\mathrm{W}_{(\mathrm{ROH})}$ & -44.3 & $-91.0(1.2)$ & $-152(2)$ & $-45.4(0.6)$ & 66.7 & 33.3 & 14.0 & 8.7 \\
& $\mathrm{ROH}_{(\mathrm{W})}$ & -51.0 & $-106.5(1.1)$ & $-182(2)$ & $-54.3(0.6)$ & 66.2 & 33.8 & 33.5 & 80.6 \\
$\mathrm{PNC}$ & $\mathrm{W}_{(\mathrm{ROH})}$ & -29.5 & $-99.3(1.7)$ & $-227(2)$ & $-67.7(0.6)$ & 59.5 & 40.5 & 143.4 & 100.5 \\
& $\mathrm{ROH}_{(\mathrm{W})}$ & -42.5 & $-78(4)$ & $-112(10)$ & $-33.4(3.0)$ & 70.0 & 30.0 & 90.7 & 38.8 \\
\hline
\end{tabular}

and in terms of entropies $\left(\% \varepsilon_{S}\right)$, were used according to the following definitions introduced by Perlovich et al. [11]:

$$
\begin{aligned}
& \% \mathcal{E}_{H}=100\left|\frac{\Delta H_{\text {spec }}^{0}}{\Delta H_{\text {non-spec }}^{0}}\right| \\
& \% \mathcal{E}_{S}=100\left|\frac{\Delta S_{\text {spec }}^{0}}{\Delta S_{\text {non-spec }}^{0}}\right|
\end{aligned}
$$

where, $\Delta H_{\text {spec }}^{0}=\Delta H_{\text {soln(solvent-i) }}^{0}-\Delta H_{\text {soln(CH) }}^{0}=\Delta H_{\text {soln(CH } \rightarrow \text { solvent-i) }}^{0}$; $\Delta H_{\text {non-spec }}^{0}=\Delta H_{\text {soln(CH) }}^{0}-\Delta H_{\text {subl }}^{0}=\Delta H_{\text {solv(CH) }}^{0}$;

$\Delta S_{\text {spec }}^{0}=\Delta S_{\text {soln(solvent-i) }}^{0}-\Delta S_{\text {soln(CH) }}^{0}=\Delta S_{\text {soln(CH } \rightarrow \text { solvent-i) }}^{0}$; and

$\Delta S_{\text {non-spec }}^{0}=\Delta S_{\text {soln(CH) }}^{0}$.

Cyclohexane was chosen as an "inert" solvent, which interacts with drug molecules solely by nonspecific interactions (dispersion forces), while the water and $\mathrm{ROH}_{(\mathrm{W})}$ interact with these drugs by specific interactions such as hydrogen bonding. Solution thermodynamics data for the drugs in $\mathrm{CH}$ presented in Table 5 were taken from Baena et al. [5].

Table 5. Thermodynamic quantities for drugs solution processes in $\mathrm{CH}$ at $25.0^{\circ} \mathrm{C}^{(a)}$.

\begin{tabular}{ccccc}
\hline Drug & $\begin{array}{c}\Delta G_{\text {soln }}^{0} / \\
\mathrm{kJ} \mathrm{mol}^{-1}\end{array}$ & $\begin{array}{c}\Delta H_{\mathrm{soln}}^{0} / \\
\mathrm{kJ} \mathrm{mol}^{-1}\end{array}$ & $\begin{array}{c}\Delta S_{\text {soln }}^{0} / \\
\mathrm{J} \mathrm{mol}^{-1} \mathrm{~K}^{-1}\end{array}$ & $\begin{array}{c}T \Delta S_{\text {soln }}^{0} / \\
\mathrm{kJ} \mathrm{mol}^{-1}\end{array}$ \\
\hline ACN 21.60 (0.04) & $69(5)$ & $159(11)$ & $47.4(3.3)$ \\
ACP $25.78(0.06)$ & $38.1(1.9)$ & $41.2(2.1)$ & $12.3(0.6)$ \\
PNC & $25.44(0.01)$ & $81.0(1.2)$ & $186(3)$ & $55.6(0.9)$ \\
\hline
\end{tabular}

(a) From Baena et al. [5].
Both the $\% \varepsilon_{H}$ and $\% \varepsilon_{S}$ values for the drugs' solvation are also presented in Table 4. These values indicate that during solution of these drugs in all mixtures studied, the specific solute-solvent interactions (hydrogen bonding, mainly) effectively affect the entropic term of free energy with respect to non-specific interactions, especially for PNC in water, although it is also significant for $\mathrm{ACP}$ in $\mathrm{ROH}_{(\mathrm{W})}$ and $\mathrm{ACN}$ in both solvents. With regard to the enthalpic term the specific solute-solvent interactions predominate for PNC in water and for ACN in both solvents $\left(\% \varepsilon_{H}>100 \%\right)$.

\section{Thermodynamics of transfer according to parti- tioning}

Table 6 summarizes the thermodynamic functions relative to transfer processes of the drugs from aqueous medium up to octanol phase taken from Baena et al. [6, 7]. The values for ACP [7] presented in the molarity scale were converted into the mole fraction scale according to procedures previously described [3].

Table 6. Thermodynamic quantities for drugs transfer processes from water to organic media at 25.0 ${ }^{\circ} \mathrm{C}$ obtained from partitioning ${ }^{(a)}$.

\begin{tabular}{ccccc}
\hline Drug & $\begin{array}{c}\Delta G_{\mathrm{w} \rightarrow 0}^{0} / \\
\mathrm{kJ} \mathrm{mol}^{-1}\end{array}$ & $\begin{array}{c}\Delta H_{\mathrm{w} \rightarrow 0}^{0} / \\
\mathrm{kJ} \mathrm{mol}^{-1}\end{array}$ & $\begin{array}{c}\Delta S_{\mathrm{w} \rightarrow 0}^{0} / \\
\mathrm{J} \mathrm{mol}^{-1} \mathrm{~K}^{-1}\end{array}$ & $\begin{array}{c}T \Delta S_{\mathrm{w} \rightarrow 0}^{0} / \\
\mathrm{kJ} \mathrm{mol}^{-1}\end{array}$ \\
\hline ACN -11.21 (0.01) & $22.4(2.6)$ & $113(13)$ & $33.7(3.9)$ \\
ACP & $-7.17(0.08)$ & $-18.9(0.6)$ & $-39.2(1.4)$ & $-11.7(0.4)$ \\
PNC $-13.10(0.04)$ & $1.5(0.2)$ & $49(6)$ & $14.6(1.8)$
\end{tabular}

${ }^{(a)}$ From Baena et al. [6, 7]. 
Both the enthalpic and entropic changes of transfer imply respectively, all the energetic requirements and the molecular randomness (increase or decrease in the molecular disorder), involved in the net transfer of the drugs from water to different organic media. In general terms, it should be considered the behavior presented in each phase, before and after the partitioning process.

Since initially the drugs are present only in water, then, it is necessary to create a cavity in the organic medium in order to accommodate the solute after the transfer process. This is an endothermic event, since an energy supply is necessary to separate the organic solvent molecules (to overcome the cohesive forces). When the solute molecules are accommodated into the organic phase an amount of energy is released due to solute-organic solvent interactions. This event would imply an entropy increase in this medium due to the respective mixing process associate.

In turn, after a certain number of solute molecules have diffused from the aqueous phase to the organic medium to reach the partitioning equilibrium, the original cavities occupied by the drug molecules in the aqueous phase have been now occupied by water molecules. This event produces an energy release due to water-water interactions. However, depending on the solute's molecular structure, it is also necessary to keep in mind the possible disruption of water-structure, that is, the water molecules organized as "icebergs" around the alkyl or aromatic groups of the drug (namely, hydrophobic effect or hydrophobic hydration). This event in particular implies an intake of energy in addition to a local entropy increase by separation of some water molecules which originally were associated among them by hydrogen bonding [12].

From Table 6 it can be observed that for ACP, the transfer process from water to $\mathrm{ROH}_{(\mathrm{W})}$ was exothermic and negentropic, whereas it was endothermic and entropic for ACN and PNC. In principle, it could be said that the obtained values in enthalpy and entropy for ACN and PCN are due mainly to disruption of water-icebergs present around the hydrocarbon groups of these drugs (methyl, ethyl, and/or phenyl groups), and on the other hand, the creation of a cavity in the organic solvent to accommodate the solute. Both events, as was already said, imply an energy intake and a disorder increase at the molecular level.

For ACP, both $\Delta H_{\mathrm{w} \rightarrow 0}^{0}$ and $\Delta S_{\mathrm{w} \rightarrow 0}^{0}$ were negative. These values could be explained in terms of a possible organization in the water-saturated octanol due to the replacement of an octanol molecule by a drug molecule. This replacement would be present in some centers conformed by two water molecules and six octanol molecules, inside the microheterogeneus structure of this water-saturated organic solvent $[4,13]$. The previous event releases energy and compensates the molecular disorder produced by the drug-organic solvent mixing process and the energy intake required in the aqueous media to separate the water molecules present around the non polar groups of this drug. On the other hand, the hydroxyl group present in ACP establishes easily $\mathrm{H}$-bonds with water, which in turn diminishes the hydrophobic hydration around the phenyl group. This particular event is different with respect to observed for ACN (around phenyl group) and PNC (around phenyl and ethyl groups). Thus, the hydrophobic hydration would be greater for both drugs respect to ACP.

Thermodynamics of solvation according to partitioning

According to Katz and Diamond [14], the values of thermodynamic functions of partitioning, $\Delta G_{w \rightarrow 0}^{0 X}, \Delta H_{w \rightarrow 0}^{0 X}$ and $\Delta S_{w \rightarrow 0}^{0 X}$, depend both upon interactions between drug and water and upon interactions between drug and organic medium. In order to obtain quantities that can be discussed solely in terms of drug-organic medium interactions, the contributions of drug-water must be removed. This can be accomplished by referring to hypothetic processes presented in Fig. 1:

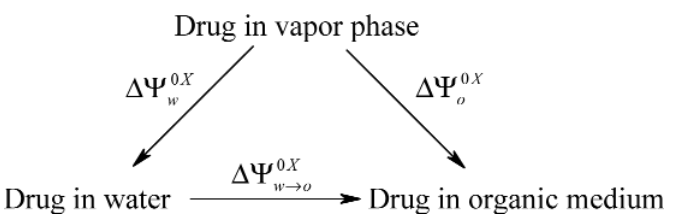

Figure 1. Transfer processes of the drugs between octanol-saturated water, water-saturated octanol, and its vapor phase. 
In which, $\Psi^{0 X}$, stands for any thermodynamic function whose change can be measured when one mole of drug is transferred between water, organic medium and the vapor phase. The term $\Delta \Psi_{w}^{0 X}$ represents the standard Gibbs free energy, enthalpy, or entropy of solvation of drug in water, while the term $\Delta \Psi_{o}^{0 X}$ represents correspondingly the standard Gibbs free energy, enthalpy, or entropy of solvation of drug in organic medium. From this, the following equations can be stated:

$$
\begin{aligned}
& \Delta \Psi_{w \rightarrow o}^{0 X}=\Psi_{o}^{0 X}-\Psi_{w}^{0 X} \\
& \Delta \Psi_{o}^{0 X}=\Psi_{o}^{0 X}-\Psi_{v}^{0 X} \\
& \Delta \Psi_{w}^{0 X}=\Psi_{w}^{0 X}-\Psi_{v}^{0 X}
\end{aligned}
$$

where, $\Psi_{v}^{0 X}$ is the respective thermodynamic value of the function in the vapor phase. The $\Delta \Psi_{w \rightarrow o}^{0 X}$ values for the drugs obtained from partitioning experiments are presented in Table 6 . On the other hand, the $\Delta \Psi_{w}^{0 X}$ values of solvation for these drugs in octanol-saturated water are presented in Table 4. From these values, the $\Delta \Psi_{o}^{0 X}$ values were calculated by means of Eq. 16:

$$
\Delta \Psi_{o}^{0 X}=\Delta \Psi_{w \rightarrow o}^{0 X}+\Delta \Psi_{w}^{0 X}
$$

Table 7 shows the standard thermodynamic functions of solvation of the drugs in $\mathrm{ROH}_{(\mathrm{W})}$ obtained considering the partitioning processes. In all cases, the $\Delta G_{\text {solv }}^{0 X}$ and $\Delta H_{\text {solv }}^{0 X}$ values are negative. These results as well as those presented in Table 4 indicate the preference of these drugs by organic media respect to its vapor phase independently of their concentrations (at saturation in solubility and highly diluted in partitioning), and also indicate that the solvation processes are enthalpy driven. In the case of $\Delta S_{\text {solv }}^{0 X}$, this function

Table 7. Thermodynamic quantities for drugs solvation processes in the aqueous and organic media at $25.0{ }^{\circ} \mathrm{C}$ obtained by considering the partitioning behavior.

\begin{tabular}{lcccc}
\hline Drug & $\begin{array}{c}\Delta G_{\text {solv }}^{0} / \\
\mathrm{kJ} \mathrm{mol}^{-1}\end{array}$ & $\begin{array}{c}\Delta H_{\text {solv }}^{0} / \\
\mathrm{kJ} \mathrm{mol}^{-1}\end{array}$ & $\begin{array}{c}\Delta S_{\text {solv }}^{0} / \\
\mathrm{J} \mathrm{mol}^{-1} \mathrm{~K}^{-1}\end{array}$ & $\begin{array}{c}T \Delta S_{\text {solv }}^{0} / \\
\mathrm{kJ} \mathrm{mol}^{-1}\end{array}$ \\
\hline ACN & -33.9 & $-46.5(3.2)$ & $-40(13)$ & $-12.0(3.9)$ \\
ACP & -51.4 & $-109.9(1.3)$ & $-192(2)$ & $-57.1(0.6)$ \\
PNC & -42.6 & $-97.8(1.7)$ & $-178(6)$ & $-53.1(1.8)$ \\
\hline
\end{tabular}

is negative which implies a diminishing in the molecular randomness by passing of drug molecules from vapor state to this liquid media. According to $\zeta_{H}$ and $\zeta_{T S}$ values, the enthalpy is the main property contributing to solvation process in all media, including the aqueous phase.

Dilution thermodynamics based on solution and partitioning

Another interesting process is the drug dilution in $\mathrm{ROH}_{(\mathrm{W})}$. The respective thermodynamic functions ( $\left.\Delta \Psi_{\text {dilution }}^{0}\right)$ are calculated according to:

$\Delta \Psi_{\text {dilution }}^{0}=\Delta \Psi_{\text {solv(Part) }}^{0}-\Delta \Psi_{\text {solv(Solub) }}^{0}$

where, $\Delta \Psi_{\text {solv(Part) }}^{0}$ and $\Delta \Psi_{\text {solv(Solub) }}^{0}$ are the thermodynamic quantities of solvation in $\mathrm{ROH}_{(\mathrm{W})}$ obtained from partitioning and solution processes (Tables 7 and 4), respectively. Table 8 shows the drugs concentrations obtained in the aqueous and organic media obtained in the partitioning equilibria. These values were calculated based on experimental details described in the literature $[6,7]$.

Table 8. Final drugs concentrations expressed in mole fraction in aqueous and organic media after partitioning.

\begin{tabular}{lcc}
\hline Drug & Aqueous & Organic \\
\hline ACN & $6.08 \times 10^{-7}$ & $5.49 \times 10^{-5}$ \\
ACP & $7.18 \times 10^{-7}$ & $1.30 \times 10^{-5}$ \\
PNC & $5.88 \times 10^{-7}$ & $1.18 \times 10^{-4}$ \\
\hline
\end{tabular}

The equilibrium solubility values in $\mathrm{ROH}_{(\mathrm{W})}$ in mole fraction at $25.0{ }^{\circ} \mathrm{C}$ for $\mathrm{ACN}$, $\mathrm{ACP}$, and PNC are 0.1248, 0.0267, and 0.0194, respectively [5]. Therefore, the drugs concentrations obtained after the hypothetic dilutions are approximately 2000-fold lower for $\mathrm{ACN}$ and ACP, and 200-fold lower for PNC. Table 9 shows the respective thermodynamic quantities for the drugs' dilution processes.

The dilution process essentially implies the diminishing in solute-solute interactions with the respective predominance of solute-solvent interactions as well as the solvent-solvent interactions. According to Table 9 the Gibbs energies of dilution were favourable for ACP and PNC $\left(\Delta G_{\text {dilut }}^{0}<0\right)$ but 
Table 9. Thermodynamic quantities for drugs dilution processes in $\mathrm{ROH}_{(\mathrm{W})}$ at $25.0^{\circ} \mathrm{C}$ obtained by considering the solubility and partitioning behavior.

\begin{tabular}{ccccc}
\hline Drug & $\begin{array}{c}\Delta G_{\text {dilut }}^{0} / \\
\mathrm{kJ} \mathrm{mol}^{-1}\end{array}$ & $\begin{array}{c}\Delta H_{\text {dilut }}^{0} / \\
\mathrm{kJ} \mathrm{mol}^{-1}\end{array}$ & $\begin{array}{c}\Delta S_{\text {dilut }}^{0} / \\
\mathrm{J} \mathrm{mol}^{-1} \mathrm{~K}^{-1}\end{array}$ & $\begin{array}{c}T \Delta S_{\text {dilut }}^{0} / \\
\mathrm{kJ} \mathrm{mol}^{-1}\end{array}$ \\
\hline ACN & $1.48(0.03)$ & $38.5(2.6)$ & $125(13)$ & $37.1(3.9)$ \\
ACP & $-0.40(0.11)$ & $-3.4(1.1)$ & $-9.6(1.5)$ & $-2.9(0.5)$ \\
PNC & $-0.07(0.07)$ & $-20(4)$ & $-66(12)$ & $-19.7(3.6)$ \\
\hline
\end{tabular}

unfavourable for $\operatorname{ACN}\left(\Delta G_{\text {dilut }}^{0}>0\right)$, whereas the respective enthalpies and entropies were negative for ACP and PNC but positive for ACN indicating enthalpy-driving for the dilution process in the case of the former drugs and entropy-driving for the last one. As was already said. $\mathrm{ROH}_{(\mathrm{W})}$ has a microheterogeneus structure centered on two molecules of water $\mathrm{H}$-bonded between them and surrounded by six octanol molecules which could be replaced by drug molecules during the solution process. Because energy must be supplied in order to overcome the solute-solute interactions during the dilution process the drugs' partial enthalpy and entropy increases as well. On the other hand, the increase in solvent-solvent interactions caused by the drug dilution process implies a decrease in the solvent partial enthalpy and entropy.

The thermodynamic values presented in Table 6 correspond to the net result obtained by considering the partial contributions of solutesolute and solvent-solvent interactions. The opposite behavior presented by ACN compared with ACP and PNC could be explained in terms of their high solubility (0.1248 in mole fraction). Because of the high drug concentration in saturation a great energy consumption is required in order to separate the drug molecules which in turn overcome the energy released by the solvent-solvent bonds established. This event also implies a great increase of the drug's partial entropy which in turn overcomes the entropy decrease presented in the solvent.

Finally, in order to clarify and understand the specific interactions presented between these drugs and $\mathrm{ROH}_{(\mathrm{W})}$, it would be very important to dispose information about UV, IR and NMR spectral data, and DSC and solution calorimetric values, among others.

\section{Conclusions}

From the previously exposed analysis, in general terms it could be concluded that these drugs have mainly a lipophilic behavior but in turn they are not certainly hydrophobic drugs. On the other hand, they are greatly solvated in the organic solvent. Although these drugs have great affinity for the $\mathrm{ROH}_{(\mathrm{W})}$ phase great differences in the possible mechanisms of transfer from the aqueous medium up to the organic solvent are found among them. These results are consequence of their successive substitutions on the phenyl ring passing from $\mathrm{ACN}$ to ACP by replacing an hydrogen atom by a hydroxyl group, and from ACP to PNC by replacing the hydroxyl group by an ethoxyl group, which in turn, changes the molar volumes and the $\mathrm{H}$-bonding properties.

\section{Received March 282008 \\ Accepted September 122008}

\section{References}

[1] L. J. Roberts II, J. D. Morrow, Goodman \& Gilman's. The Pharmacological Basis of Therapeutics, 10th edn., Hardman, J. G.; Limbird, L. E.; Gilman, A. G., eds., McGraw-Hill, New York, 2001, ch. 27.

[2] G. L. Perlovich, T. V. Volkova, A. Bauer-Brandl, J Pharm Sci. 95(10) (2006) 2158.

[3] C. P. Mora, F. Martínez, J. Chem. Eng. Data 52(5) (2007) 1933.

[4] J. Sangster, Octanol-Water Partition Coefficients: Fundamentals and Physical Chemistry, John Wiley \& Sons, Chichester, 1997.

[5] Y. Baena, J. A. Pinzón, H. Barbosa, F. Martínez, Phys. Chem. Liquids 42(6) (2004) 603.

[6] Y. Baena, J. Pinzón, H. Barbosa, F. Martínez, Acta Pharm. 55(2) (2005) 195.

[7] Y. Baena, J. Pinzón, H. Barbosa, F. Martínez, Rev. Bras. Cienc. Farm. 40(3) (2004) 413.

[8] J. M. Diamond, Y. Katz, J. Membrane Biol. 17(1) (1974) 121. [9] S. Budavari, M. J. O’Neil, A. Smith, P. E. Heckelman, J. R. Obenchain Jr, J. A. R. Gallipeau, M. A. D’Arecea, The Merck Index, An Encyclopedia of Chemicals, Drugs, and Biologicals, 13th edn., Merck \& Co., Inc., Whitehouse Station, NJ, 2001.

[10] C. P. Mora, F. Martínez, Fluid Phase Equilibria 255 (2007) 70.

[11] G. L. Perlovich, S. V. Kurkov, A. N. Kinchin, A. BauerBrandl, Eur. J. Pharm. Biopharm. 57(2) (2004) 411.

[12] C. Tanford, The Hydrophobic Effect: Formation of Micelles and Biological Membranes, John Wiley \& Sons, New York, 1973.

[13] C. P. Mora, H. R. Lozano, F. Martínez, Rev. Bras. Cienc. Farm. 41(1) (2005) 13.

[14] Y. Katz, J. M. Diamond, J. Membrane Biol. 17(1) (1974) 101. 